\title{
A Study on the Participation Motivation of "Send and Receive Network Red Envelopes"
}

\author{
SUN Yuan
}

Education School, Jianghan University, Wuhan, China

\author{
273234540@qq.com
}

\begin{abstract}
Keywords Network red envelopes, Sense of achievement, Economic interests, Conformity psychology, Social contact psychology.
\end{abstract}

\begin{abstract}
With the popularity of smart phones and the development of Internet technology, the phenomenon of sending and receiving network red envelopes has penetrated into people's daily life, and the study is to explore theparticipation motivation of "send and receive network red envelopes" phenomenon, so that we can treat it in a reasonable manner. Firstly, through literature analysisand open questionnaire survey, we can understand the participation motivation of sending and receiving network red envelopes, and construct the theoretical model, then a questionnaire survey was conducted among 537 subjects who had used network red envelopes, and exploratory factor analysis and confirmatory factor analysis were conducted using SPSS17.0 and AMOS24.0. The model construction is reasonable. Gamesentertainment, obtaining a sense of achievement, obtaining economic interests, conformity psychology and social contact psychology are 5 factors that influence the behavior of sending and receiving network red envelopes. The reliability and validity of the participation motivation questionnaire of "send and receive network red envelopes"basically meet the requirements of psychometrics.
\end{abstract}

\section{“收发网络红包”的参与动机研究 \\ 孙远 \\ 江汉大学教育学院, 湖北武汉, 中国 \\ 273234540@qq.com}

关键词: 网络红包; 成就感; 经济利益; 从众心理; 社交心理

中文摘要. 随着智能手机的普及和互联网技术的发展, 收发网络红包的现象已经深入到人们 的日常生活中，本研究探讨 “收发网络红包”的参与动机，以让我们用合理的态度对待网络红 包。先通过文献分析、开放式问卷调查等方法了解收发网络红包的参与动机, 建构理论模型, 之后采用问卷调查的方法对537名被调查者进行调查, 用SPSS17.0和AMOS24.0对数据进行探 索性因素分析和验证性因素分析。模型建构比较合理, 收发网络红包的参与动机有游戏娱乐、 获得成就感、获得经济利益、从众心理和社交心理5个因素组成。自编的“收发网络红包”参与 动机问卷的信度和效度基本上符合心理测量学的要求。

\section{1.引言}

近年来, 随着智能手机的快速普及和移动互联网技术的发展, 微信、微博、 $\mathrm{QQ}$ 等移动端 社交软件让人与人之间的联系更加便捷密切。支付巨头“微信支付”在2014年1月正式推出“微 信红包”，从此网络红包市场正式开始。如今微博、微信、QQ、支付宝等都推出了“抢红包” 
活动, 并且不仅仅只限于节假日, 目前抢红包活动已经渗透到日常生活的每分每秒当中了 ${ }^{[1]}$ 。 红包又称为压岁钱, 是在春节时年长者用红色的纸将钱包起来给晚辈的一种礼物, 红包中寄 托着的是期望, 也是祝福, 希望晚辈在新的一年中顺风顺水。和传统红包相比, 网络红包可 以是长辈给晚辈，也可以是晚辈给长辈、同辈之间，时间和地点都比较随意。网络红包按照 发放红包主体的不同可以分为两类，一类发红包的主体是商户，一类是个人。本课题主要研 究个人间网络红包, 对商业红包不做研究, 即这里的个人间网络红包主要是指由亲戚朋友、 同事、他人等通过网络方式发放的红包, 不包括通过红包形式转账、还款和商品支付等情况。

网络红包的广泛运用，使得很多个体都参与其中，对个体的心理和行为都产生了较大的 影响。为了让我们用合理的态度对待网络红包, 使网络红包产生最大化效益, 增强个体和谐 的人际关系，本研究探讨有哪些因素影响个体收发网络红包的参与动机。

\section{2.理论模型}

参与动机是指由内在或外在需要引起的, 个体通过参与某项活动来满足各种需要的特殊 心理状态和意愿 ${ }^{[2]}$ 。对于游戏的参与行为最认可的解释是自我决定理论 ${ }^{[3]}$ 。自我决定理论将个 体参与动机分为内部动机、外部动机和内部化动机三种类型, 内部动机是指参与某项活动本 身带来的愉悦与满足感 ${ }^{[4]}$; 外部动机是指参与相关活动从而带来一些额外的利益回报; 而内 部化动机则是指由外部因素引起从而逐渐内化的调节过程, 比如感到的胜利感和竞争感等。

\section{1 游戏娱乐心理}

“抢红包”活动创造了一个新的娱乐场景 ${ }^{[5]}$, 因为它具有游戏的基本特性: 竞争性、变化 性和方向性 ${ }^{[6]}$ 。竞争性表现在参与“收发网络红包”活动中抢到或者发出一个相比较于其他人 金额高的红包。变化性则是体现在每次点开红包都不知道自己能够抢到的金额，从而产生一 种期待心理。方向性表现在大家都期望着自己能够得到最后的“胜利”，即获得金额最高，比 如“拼手气红包”则是采用随机算法，使得用户的每一次“抢”红包行为充满未知性 [7]。在抢发红 包时可以感受到参与游戏的乐趣:放松心情、愉悦身心。所以, 在“收发网络红包”活动中游戏 娱乐心理可能是一个影响因素。

\section{2 获得成就感}

个体在抢红包过程中，由于抢到的红包金额数是随机的，当个体抢到了最高金额数的红 包时，会产生一种成就感，因为获得最高金额数的红包说明个体的运气相比较于其他人好。 对于发红包的人也有相同的心理，在QQ红包中，有一个“口令红包”，即发红包个体将红包设 置一些语句, 只有抢红包者在对话框中输入发红包人所设置的语句才能获得红包, 在这种情 况下, 个体可以产生一种能够控制他人说话内容的成就感。因此获得成就感也可能是影响收 发网络红包的一个因素。

\section{3 获得经济利益}

“收发网络红包”活动本身就带有一定的金钱交易。根据认识评价理论，物质奖励对内在 动机的维持有显著影响 ${ }^{8]}$ 。在虚拟金钱奖赏下，前次决策结果对个体当前的风险决策行为没 有显著的影响 ${ }^{[9]}$, 在抢红包的活动中也如此, 前次抢红包结果对下一次抢红包行为的影响效 果并不明显，导致个体为了获得经济利益会不断参与抢红包的活动。

\section{4 从众心理}

对于消费者的从众行为, 从营销学的角度解释, 认为消费者为了获得群体的认同、符合 群体的期望, 会采取与群体其他成员相似的行为。宋官东则是从社会心理学角度将从众认为 是主体因客体的影响, 从而产生与客体相一致的态度或行为 ${ }^{[10]}$ 。在QQ或微信群中，看到他人 收发红包，为了与大家保持一致也会采取相同的行为，这就是从众心理的影响。 


\section{5 社交心理}

互联网的出现使得人与人之间的联系更加密切了，同样也对人际间的关系产生了巨大影 响。在微信中, 圈内好友通过微信红包达到相互交流的目的 ${ }^{[11]}$ 。红包就是互联网世界所创造 出的人与人之间的一种礼物，一种虚拟化的馈赠 ${ }^{[12]}$ 。网络红包除了时间成本低，本身也是一 种人际间的交往方式, 拉近人与人之间情感的活动。我发你抢的这种互动, 也表明个体希望 在社交群体中能够有所表现，以及和其他个体间相互交流的期望。随着生活节奏的加快，人 际间的关系越来越淡, 人情冷漠现象也逐渐渗透到社会各个层次中, 所以人际关系是需要每 个人细心呵护和维护的。加强人际关系通常由两种方法：一种是经历与合作，共同参与某个 活动, 另一种是沟通, 以文字、语音、视频、物品等为媒介进行沟通 ${ }^{[13]}$ 。网络红包是沟通的 一种便捷有效的方法, 并通过游戏化的金钱奖励, 将两人之间的关系迅速拉近, 加深人际关 系。个人间网络红包是以真金白银为心意, 代替各种无聊的祝福语, 纵使红包的数额可能不 是很大，这种实在物质奖励也足以表达发红包者的心意。

基于以上的理论基础, 本研究提出了收发网络红包参与动机的五因素模型：游戏娱乐、 获得成就感、获得经济利益、从众心理、社交心理。

\section{3. 研究过程}

\section{1 问卷编制}

先进行一次开放性问卷调查, 问卷共有8道题目, 不设具体答案, 由被调查者进行开放式 回答。根据开放性问卷的整理结果和理论模型, 编写出预调查问卷。共有33道题目, 5 个因素, 采用李克特的5 点计分法计分, “非常不符合”记1 分, “比较不符合”记2 分, “不确定”记3分, “比较符合”记4 分, “非常符合”记5 分。问卷共计发放 208 份, 有效问卷 181 份, 有效率为 $87 \%$ 。 采用SPSS17.0软件进行统计分析。

根据数据分析可知，每个因素的Cronbach’s Alpha系数均在0.624-0.787之间，总体信度为 0.793。因此, 该问卷具有良好的信度指标, 即有较好的稳定性和可靠性。

\section{2 探索性因素分析}

根据Comery提出的KMO和Baratlett球形检验的条件 ${ }^{[14]}, \mathrm{KMO}>0.7, \mathrm{p}=0.000$, 表明收集 的样本数据适合做因素分析。

依据特征值大于 1 的原则提取因子，共提取 5 个因子，旋转因子负荷矩阵见表 1 。 5 个公共 因子的累计解释方差为 $81.28 \%$ 。

表1 预调查旋转因子负荷矩阵

\begin{tabular}{|c|c|c|c|c|c|}
\hline & 1 & 2 & 3 & 4 & 5 \\
\hline E1 & & & 0.475 & & \\
\hline E2 & & & 0.742 & 0.480 & \\
\hline E3 & & & 0.803 & & \\
\hline E4 & & & 0.675 & & \\
\hline E5 & & & 0.814 & & \\
\hline E6 & & & 0.692 & & \\
\hline E7 & & & 0.659 & & \\
\hline $\mathrm{F} 1$ & & 0.402 & & & \\
\hline $\mathrm{F} 2$ & & 0.601 & & & \\
\hline F3 & & 0.814 & & & \\
\hline F4 & & 0.574 & & & \\
\hline F5 & & 0.749 & & & \\
\hline
\end{tabular}


0.714

$\begin{array}{ll}\text { P1 } & 0.459 \\ \text { P2 } & 0.692 \\ \text { P3 } & 0.522 \\ \text { P4 } & 0.636 \\ \text { P5 } & 0.686 \\ \text { P6 } & 0.583 \\ \text { P7 } & 0.745\end{array}$

C1

$\begin{array}{cc} & 0.550 \\ & 0.527 \\ & 0.514 \\ 0.415 \quad 0.765 \\ & 0.531 \\ & 0.602\end{array}$

0.408

$\mathrm{C} 2$

C3

$\mathrm{C} 4$

0.644

S1

0.438

S2

0.551

S4

0.689

S5

0.585

S6

0.628

S7

0.539

根据表1中数据可知, E1分数低于 $0.5, \mathrm{E} 2$ 横跨两个域, 综合考量该因素的各项指标, 还 是将这两个题项删除, 即该因素将保留在E3、E4、E5、E6和E7共计5个题项上, 命名为“游戏 娱乐”; F1 题项分数低于 0.5 , 综合考量该因素的各项指标, 还是将这个题项删除, 即该因素 将保留在F2、F3、F4、F5和F6共计5个题项上, 命名为“获得成就感”; P1和P3两个题项都有 跨域出现, 综合考量该因素的各项指标, 还是将这两个题项删除, 即该因素将保留在P2、P4、 P5、P6和P7共计5个题项上，命名为“获得经济利益”; C5 题项出现横跨两个域现象，综合考 量该因素的各项指标，还是将这个题项删除，即该因素将保留在 C1、C2、C3、C4和C6共计5 个题项上, 命名为“从众心理”; S2 题项分数低于 0.5 , 综合考量该因素的各项指标, 还是将这 个题项删除, 即该因素保留在S1、S3、S4、S5、S6和S7共计6个题项上，命名为“社交心理”。

\section{3验证性因素分析}

在探索性因素分析的结果基础上, 删减了7道题目, 修改了意义不明、敏感性高、有歧义 的题目, 形成正式的调查问卷, 含有 26 个题项。正式问卷通过网络途径来收集数据, 共计发 放问卷 410 份, 有效问卷 356 份, 有效率为 $86.82 \%$ 。采用AMOS24.0软件对数据进行统计处理。

利于AMOS24.0软件构建结构方程模型, 在结构方程模型中主要含有三类变量: 潜在变量、 观察变量和误差变量。潜在变量是指那些不能够直接测量的变量, 一般是设计模型中的自变 量和因变量, 用椭圆形表示。观察变量是可以测量的变量, 一般是模型中自变量和因变量所 代表的测量项, 用方框表示。误差变量是自变量和因变量存在的误差。本研究中, 共有游戏 娱乐、获得成就感、获得经济利益、从众心理、社交心理 5 个潜在变量, 观察变量共计有 26 个，在AMOS24.0中建构模型如图1。

利于最大似然法对模型中的相对卡方值 $(\mathrm{CMIN} / \mathrm{DF})$ 、拟合优度指数(GFI)、规范拟合指数 (NFI)、比较拟合指数 $(\mathrm{CFI})$ 和近似误差均方根(RMSEA) 共 5 个常用拟合度指标进行评估。修正 前该模型拟合度基本上可用, 根据数据提示信息进行修正, 删除第 23 题, 保留其他 25 题, 对 剩下的 25 题再次进行验证性因素分析, 发现各个模型的拟合度有所改善, 达到了要求, 具体 见表2。 
因此修正后的模型是可以接受的，5因素结构得到了基本验证，可以作为研究“收发网络 红包”参与动机的测量工具。

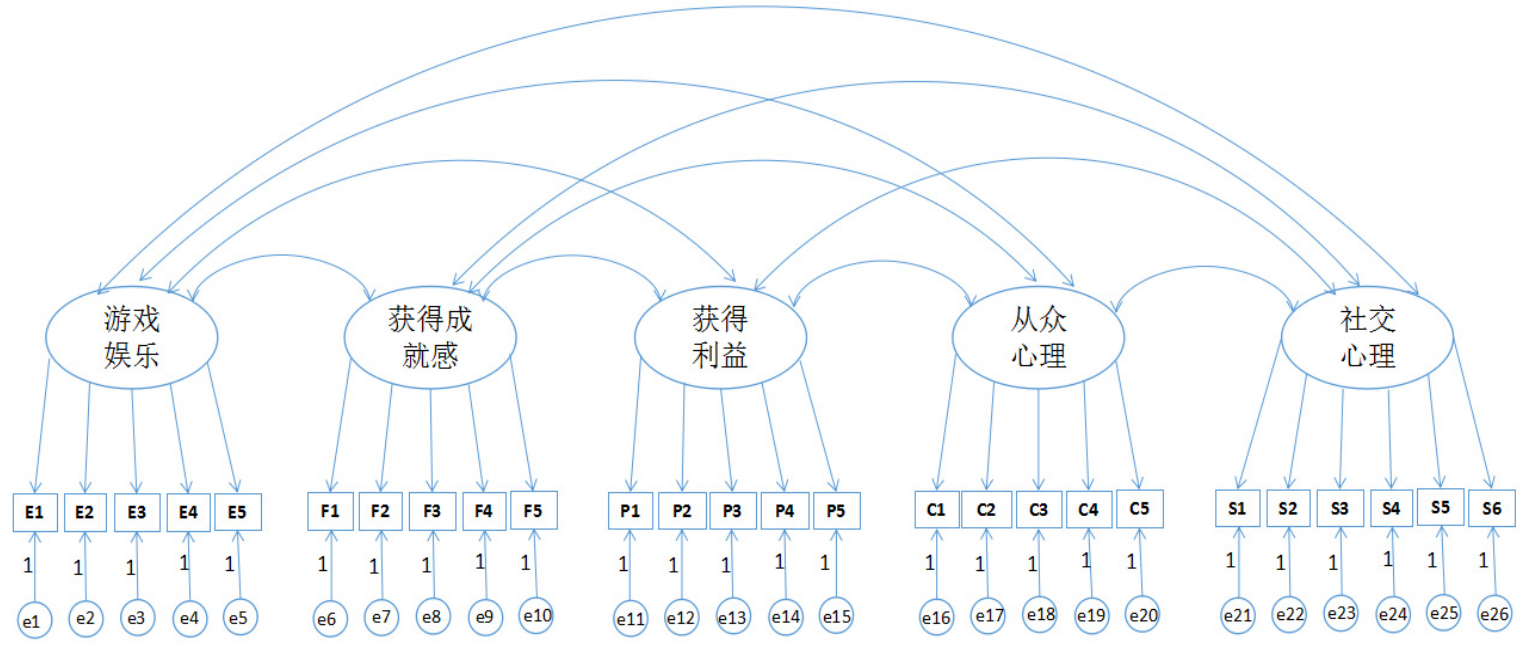

图1消费者“收发网络红包”活动参与动机影响因素结构模型

表2 设计模型拟合度指数

\begin{tabular}{cccccc}
\hline 拟合指标 & CMIN/DF & GFI & NFI & CFI & RMSEA \\
可接受值 & $<2$ & $>0.90$ & $>0.90$ & $>0.90$ & $<0.05$ \\
修正前 & 2.125 & 0.867 & 0.900 & 0.889 & 0.053 \\
修正后 & 1.95 & 0.945 & 0.926 & 0.901 & 0.046 \\
\hline
\end{tabular}

\section{4. 结论}

个体“收发网络红包”的参与动机是由游戏娱乐、获得成就感、获得利益、从众心理和社 交心理构成的。自编的“收发网络红包”参与动机问卷基本上符合心理测量学的要求。

收发网络红包现象已经深入我们的日常生活, 我们可以好好利用它以服务于我们的工作、 生活。在紧张的学习、工作之余, 可以通过收发红包游戏来放松愉悦自己。比如说在微信群, 第一个人开始发红包，之后获得“手气最佳” 者也接着发一个红包出来，如此循环下去，这样 的一个“小游戏”可以让用大家的注意力短暂集中在“抢红包”中，从而达到放松娱乐的目的。 利用获得经济利益和成就感的驱动, 当我们需要别人协助完成某项任务时, 可以把任务放在 群中，同时发一个较大金额的红包，这样可以促使他人帮忙，加快任务的完成。

\section{5. 结束语}

本文针对近年来流行的网络红包现象，研究个体收发网络红包的参与动机，主要通过问 卷调查的形式, 采用因素分析、结构方程建模的方法进行。了解了收发网络红包的参与动机, 在需要的时候，可以利用网络红包使人们的生活更加便捷、有意义和充满乐趣。

本研究的局限在于研究的样本多数集中在18-30岁, 后续研究还需要扩大样本的覆盖范 围。收红包和发红包的参与动机可能是不同的, 但是本研究没有做以区分, 后续研究可以分 开来进行更深入地探讨。

\section{致谢}

本文为湖北省教育厅人文社会科学研究项目“微时代背景下大学生人际交往模式及辅导 研究”(16G089)的阶段性成果之一。 


\section{References}

[1] http://tech.sina.com.cn/i/2015-02-25/doc-icczmvun6261641.shtml/2015-02-25

[2] Deci EL. Effects of externally mediated rewards on intrinsic motivation, Journal of Applied Social Psychology, vol. 28(15), pp. 1429-1464, 1971.

[3] Ryan RM, Rigby CS, Przbylski A. The motivational pull of video games: A self-determination theory approach, Motivation and Emotion, vol. 30(4), pp.344-360, 2006.

[4] Deci EL, Ryan RM. The support of autonomy and the control of behavior, Journal of Personality \& Social Psychology, vol. 53(6), pp.1047-1037, 1987.

[5] Li Jieke, Looking at the WeChat red envelope from the Internet thinking marketing, Marketing Research, vol. 3, pp. 37-38, 2014.

[6] Wang Bo, Research on psychological factors in game design, Wuhan University of Technology Master thesis, 2006.

[7] Xu Qi, "New" Thinking of "WeChat Red Packet". China Media Technology, vol. 2, pp. 2730, 2014.

[8] Feng Zhuqing, Ge Yan, The Undermine Effect of Material Reward to Intrinsic Motivation, Advances in Psychological Science, vol. 22(4), pp.685-692,2014.

[9] Xu Sihua, Fang Zhuo, Rao Hengyi, Real or Hypothetical Monetary Rewards Modulates Risk Taking Behavior. Acta Psychologica Sinica, vol. 45(8), pp. 874-886, 2013.

[10] Song Guandong, New theory of congregation, Journal of Psychological Science, vol. 28(5), pp. 1174-1178, 2005.

[11] Shen Sida, Chen Yong, A Probe into the Influence of WeChat Red Packet Propagation on Interpersonal Relationship, Public Communication of Science \& Technology, vol. 8, pp. 110-111, 2015.

[12] Xie Yue, The Change on Traditional Social Relationship by Discussing the Red Packets on Wechat, Journal of Tangshan Normal University, vol. 37(4), pp.78-81, 2015.

[13] Wang Huan, Wang Sujun, Analysis of the Influence of WeChat Red Packet on the Construction of Interpersonal Relationship, Southeast Communication, vol. 4, pp. 64-67, 2016.

[14] Comrey, A. Common methodological problems in factor analytic studies, Journal of Clinical and Consulting Psychology, vol. 46(4), pp. 648-659, 1978. 